\title{
Quantum fluctuations in anisotropic triangular lattices with ferro- and antiferromagnetic exchange
}

\author{
Burkhard Schmidt and Peter Thalmeier \\ Max-Planck-Institut für Chemische Physik fester Stoffe, 01187 Dresden, Germany
}

\begin{abstract}
The Heisenberg model on a triangular lattice is a prime example for a geometrically frustrated spin system. However most experimentally accessible compounds have spatially anisotropic exchange interactions. As a function of this anisotropy, ground states with different magnetic properties can be realized. Motivated by recent experimental findings on $\mathrm{Cs}_{2} \mathrm{CuCl}_{4-x} \mathrm{Br}_{x}$, we discuss the full phase diagram of the anisotropic model with two exchange constants $J_{1}$ and $J_{2}$, including possible ferromagnetic exchange. Furthermore a comparison with the related square lattice model is carried out. We discuss the zero-temperature phase diagram, ordering vector, ground-state energy, and ordered moment on a classical level and investigate the effect of quantum fluctuations within the framework of spin-wave theory. The field dependence of the ordered moment is shown to be nonmonotonic with field and control parameter.
\end{abstract}

PACS numbers: 75.10.Jm, 75.30.Cr, 75.30.Ds

\section{INTRODUCTION}

The 2D isotropic triangular Heisenberg antiferromagnet (HAF) is the most simple spin model that exhibits geometric frustration when only next neighbor (n.n) coupling is considered. In fact the triangular lattice is the only Bravais lattice in 2D which is geometrically frustrated. Classically (i.e., for large $\mathrm{S}$ ) the ground state of this model is the noncollinear $120^{\circ}$ structure corresponding to a commensurate spiral order. For a quantum spin $S=1 / 2$ the interplay of quantum fluctuations and frustration leads to anomalous ground state properties and low energy excitations. This has been studied for considerable time by analytical [1, 2] as well as numerical [3,-5] methods. It was finally concluded that the magnetic order is stable even in the quantum case, albeit with strongly reduced ordered (staggered) moment $m_{Q} /\left(\mu_{B} S\right) \approx 0.41$. This reduction may quantitatively be understood as the effect of zero-point fluctuations in linear spin wave (LSW) theory. It not only affects the moment size but also the ground state energy, magnetization and homogeneous susceptibility. These corrections are formally of the order $(1 / \mathrm{S})$. It was found that $(1 / \mathrm{S})$ corrections also strongly modify the LSW (classical) dispersion itself [6], leading to an overall band width reduction, and additional (roton-type) minimum and peculiar life-time effects [7]. This agrees with the results of high-temperature series expansions [8].

While the theoretical picture is fairly well established, there is, however, no good material candidate for the isotropic $\mathrm{S}=1 / 2$ quantum $\mathrm{HAF}$ on the triangular lattice. The systems studied sofar have considerable interlayer-exchange and inplane anisotropies [9], in particular for the most well studied $\mathrm{Cs}_{2} \mathrm{CuCl}_{4}[10,-12]$ and $\mathrm{Cs}_{2} \mathrm{CuBr}_{4}[12,13]$ compounds and their substitutional series $\mathrm{Cs}_{2} \mathrm{CuCl}_{4-x} \mathrm{Br}_{x}[14]$. Therefore it is not sufficient to just focus on the isotropic case. A related observation is made for the square lattice (interaction-) frustrated $J_{1}-\mathrm{J}_{2}$ HAF (see e.g. Ref. 15 and references cited therein). Most of the layered vanadium oxide compounds corresponding to this model have strongly different exchange constants with $J_{2}$ being actually ferromagnetic while $J_{1}$ is antiferromagnetic. This is not only a superficial analogy because, as we discuss below the anisotropic triangular HAF is equivalent to the

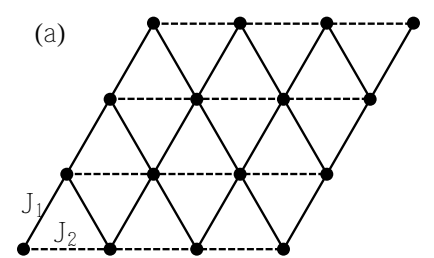

(b)

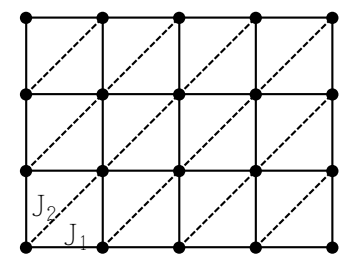

FIG. 1. (a) anisotropic triangular lattice with exchange constants $J_{1}$ (solid bonds) and $J_{2}$ (dashed bonds) which is topologically equivalent to a square lattice with one additional set of diagonal bonds (b).

square lattice $J_{1}-J_{2}$ HAF model with one $J_{2}$ bond cut. Therefore, throughout our work we will emphasize similarities and differences of both models.

Due to this predominance of anisotropic exchange materials it is highly useful not only to consider the isotropic triangular HAF but to investigate the full phase diagram of the anisotropic model, in particular because the substitutional series $\mathrm{Cs}_{2} \mathrm{CuCl}_{4-x} \mathrm{Br}_{x}$ may allow a tuning of the anisotropy ratio within a certain range [14]. It is a further interesting aspect of this model that by tuning a single control parameter one may go from square lattice HAF $(\square)$ via isotropic triangular $(\triangle)$ to quasi-1D spin chain $(\|)$ system. There exist several analytical [16-20] and numerical [21-24] investigations of the triangular model and its generalization [25] but only for the case when both anisotropic exchange constants $\mathbf{J}_{1}$ and $\mathbf{J}_{2}$ are antiferromagnetic.

Here we present an analysis of the general triangular exchange model without restriction to $\mathrm{J}_{1}$ and $\mathrm{J}_{2}$ (or the anisotropy angle $\left.\phi=\tan ^{-1}\left(J_{2} / J_{1}\right)\right)$. Furthermore we investigate magnetization and field dependence of ordered moment in the full range of the anisotropy parameter. We use the linear spin wave (LSW) theory in an external field as starting point of our analysis, although one must keep in mind that this method is strictly not applicable in the quasi-1D and AF/spiral boundary regions of the phase diagram. Our emphasis is to derive the systematic trend of $(1 / \mathrm{S})$ quantum corrections in ground state energy, ordering vector, staggered moment and magnetization as function of the anisotropy angle $\phi$ in the whole range $-\pi \leq \phi \leq \pi$. This should be very useful information for the interpretation of real anisotropic triangular magnets and their 
location in the phase diagram as it has been for the square lattice cases, in particular for layered $\mathrm{V}$ oxides [26, 27] and also for the quasi-1D system $\mathrm{CsCuCl}_{3}$ with in-plane triangular structure [28]. Here we do not yet consider the $(1 / \mathrm{S})$ corrections to the excitation spectrum itself as function of $\phi$, as was done for the isotropic $\phi=\pi / 4$ in $\operatorname{Ref} 7$, this is left for a later investigation.

\section{MODEL HAMILTONIAN}

The model spin Hamiltonian for the anisotropic triangular lattice is given by

$$
\mathscr{H}=\sum_{\langle i j\rangle} \mathbf{S}_{i} J_{i j} \mathbf{S}_{j}-g \mu_{\mathrm{B}} \mu_{0} \mathbf{H} \sum_{i} \mathbf{S}_{i}
$$

where the sum in the first term extends over bonds $\langle i j\rangle$ connecting sites $i$ and $j$. We assume an interaction in the form of a uniaxial tensor in spin space defined by

$$
J_{i j}=\operatorname{diag}\left(J_{i j}^{\perp}, J_{i j}^{\perp}, J_{i j}^{z}\right),
$$

and the applied magnetic field $\mathbf{H}$ points into the $z$ direction defined by the anisotropy above. Figure 1 illustrates the spatial structure of $\mathscr{H}$, and we set

$$
J_{i j}=\left\{\begin{array}{l}
J_{1} \text { if } \mathbf{R}_{j}=\mathbf{R}_{i} \pm \frac{1}{2}\left(\mathbf{e}_{x} \pm \sqrt{3} \mathbf{e}_{y}\right) \\
J_{2} \text { if } \mathbf{R}_{j}=\mathbf{R}_{i} \pm \mathbf{e}_{x}
\end{array},\right.
$$

measuring distances in units of the lattice constants. For simplicity we omit the directional indices $\perp$ and $z$ in the following, unless otherwise noted.

The Fourier transform of the exchange integral for the lattice with $N$ sites then reads

$$
\begin{aligned}
J(\mathbf{q}) & =\frac{1}{N} \sum_{\langle i j\rangle} J_{i j} \mathrm{e}^{-\mathrm{iq}\left(\mathbf{R}_{i}-\mathbf{R}_{j}\right)}=\frac{1}{2} \sum_{n} J_{i i+n} \mathrm{e}^{-\mathrm{iq} \mathbf{R}_{n}} \\
& =2 J_{1} \cos \frac{q_{x}}{2} \cos \frac{\sqrt{3}}{2} q_{y}+J_{2} \cos q_{x},
\end{aligned}
$$

where the last sum runs over all bonds $n$ connecting an arbitrary but fixed site $i$ with its six neighbors. Alternatively, we can regard the lattice as an isotropic square lattice with one additional diagonal bond as shown in Fig. 1p. The mapping corresponds to a coordinate transformation in crystal momentum space

$$
q_{x} \rightarrow k_{x}+k_{y}, \quad q_{y} \rightarrow \frac{1}{\sqrt{3}}\left(k_{x}-k_{y}\right),
$$

and the exchange Fourier transform acquires the form

$$
J(\mathbf{k})=J_{1}\left(\cos k_{x}+\cos k_{y}\right)+J_{2} \cos \left(k_{x}+k_{y}\right) .
$$

We will use the symbols $q, Q$ for the triangular lattice coordinates, and use $k, K$ for the square lattice coordinates.

In order to discuss the full phase diagram of the model, we introduce a anisotropy angle $\phi$ and an overall energy scale $J_{\mathrm{c}}$

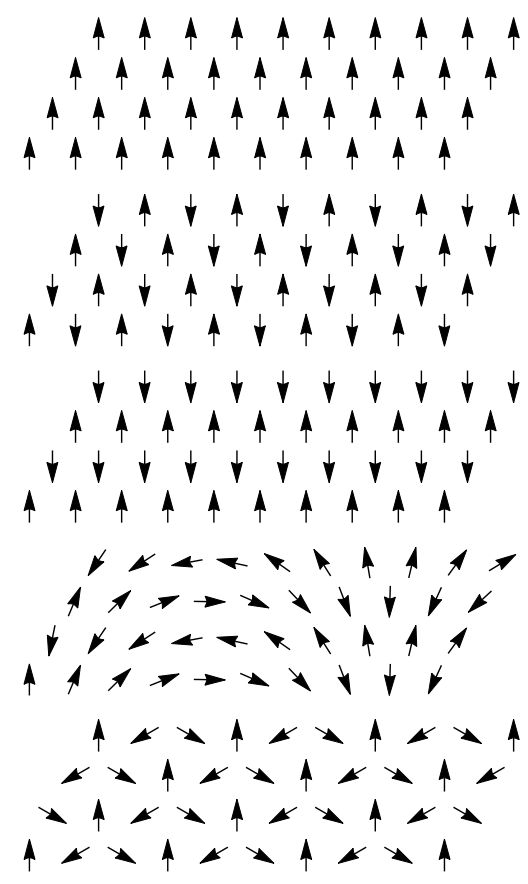

FIG. 2. Moment patterns of the ordered states, from top to bottom: Ferromagnet, columnar antiferromagnet, antiferromagnet, spiral with $J_{2} / J_{1}=1 / 2+0.01$, special case: 120 -degree-structure for $J_{2}=J_{1}>0$ (isotropic AF exchange).

defined through

$$
\begin{array}{cl}
J_{1}=J_{\mathrm{c}} \cos \phi, & J_{2}=J_{\mathrm{c}} \sin \phi, \\
J_{c}=\sqrt{J_{1}^{2}+J_{2}^{2}}, & \phi=\tan ^{-1}\left(\frac{J_{2}}{J_{1}}\right) .
\end{array}
$$

This parameterization allows for an interpolation between important geometrical limiting cases, namely the square-lattice Néel antiferromagnet $\left(J_{2}=0\right.$ or $\left.\phi=0\right)$, the isotropic triangular antiferromagnet $\left(J_{2}=J_{1}\right.$ or $\left.\phi=\pi / 4\right)$, the antiferromagnetic chain $\left(J_{1}=0\right.$ or $\left.\phi=\pi / 2\right)$, and their ferromagnetic counterparts. The following analysis of the model with the parameterization introduced here closely follows the general concept presented in [15].

\section{CLASSICAL GROUND STATES}

On each site $i$, we introduce a local coordinate system where the local $z^{\prime}$ axis is oriented parallel to the local magnetic moment, which we regard as a classical vector. The global $z$ axis is defined by the direction of the magnetic field $\mathbf{H}$. Ignoring anisotropies, the spins undergo a spin-flop transition for arbitrary small fields. We can parameterize them with a canting angle $\Theta$ relative to the field direction and an ordering vector $\mathbf{Q}$ in the $x y$ plane perpendicular to the field direction. The value $\Theta=\pi / 2$ corresponds to the state with vanishing magnetic field (arbitrary global $z$ axis), and $\Theta=0$ corresponds to the fully polarized state at the saturation field. 
TABLE I. Classical ground states: Ordering vectors, energies, conditions, range of anisotropy angle. For each phase the possible equivalent wave vectors in the first BZ that produce the same spin structure are given.

\begin{tabular}{|c|c|c|c|c|}
\hline phase & classical ordering vectors $\mathbf{Q}$ & $E_{\mathrm{cl}} /\left(N S^{2}\right)$ & conditions & range \\
\hline ferromagnet & $\begin{array}{l}0 \\
\left( \pm 2 \pi, \pm \frac{2 \pi}{\sqrt{3}}\right)\end{array}$ & $2 J_{1}+J_{2}$ & $J_{1} \leq 0 \wedge \frac{J_{2}}{\left|J_{1}\right|} \leq \frac{1}{2}$ & $-\pi-\tan ^{-1}\left(\frac{1}{2}\right) \leq \phi \leq-\frac{\pi}{2}$ \\
\hline columnar AF & $\left( \pm \pi, \pm \frac{\pi}{\sqrt{3}}\right)$ & $-J_{2}$ & - & - \\
\hline antiferromagnet & $\begin{array}{l}( \pm 2 \pi, 0) \\
\left(0, \pm \frac{2 \pi}{\sqrt{3}}\right)\end{array}$ & $-2 J_{1}+J_{2}$ & $J_{1} \geq 0 \wedge \frac{J_{2}}{J_{1}} \leq \frac{1}{2}$ & $-\frac{\pi}{2} \leq \phi \leq \tan ^{-1}\left(\frac{1}{2}\right)$ \\
\hline spiral & $\left\{\begin{array}{l}\left. \pm 2 \tan ^{-1}\left(\sqrt{4\left(\frac{J_{2}}{J_{1}}\right)^{2}-1}\right), 0\right) \\
\left. \pm 2 \tan ^{-1}\left(\sqrt{4\left(\frac{J_{2}}{J_{1}}\right)^{2}-1}\right), \pm \frac{2 \pi}{\sqrt{3}}\right)\end{array}\right.$ & $-J_{2}\left[1+\frac{1}{2}\left(\frac{J_{1}}{J_{2}}\right)^{2}\right]$ & $J_{2} \geq 0 \wedge \frac{J_{2}}{\left|J_{1}\right|} \geq \frac{1}{2}$ & $\tan ^{-1}\left(\frac{1}{2}\right) \leq \phi \leq \pi-\tan ^{-1}\left(\frac{1}{2}\right)$ \\
\hline isotropic triangular $\mathrm{AF}$ & $\begin{array}{l}\left( \pm \frac{4 \pi}{3}, 0\right) \\
\left( \pm \frac{2 \pi}{3}, \pm \frac{2 \pi}{\sqrt{3}}\right)\end{array}$ & $-\frac{3}{2} J_{1}$ & $J_{1}=J_{2}>0$ & $\phi=\frac{\pi}{4}$ \\
\hline
\end{tabular}
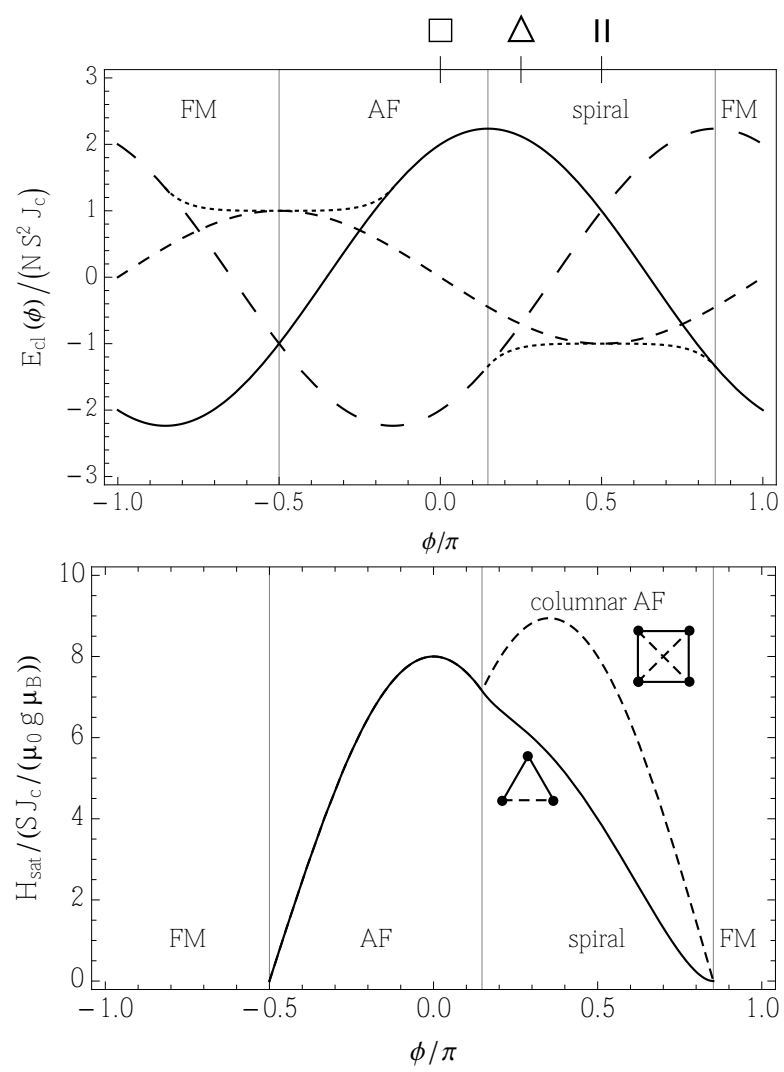

FIG. 3. Top: Dependence of the classical energies listed in Table $\mathrm{I}$ on the anisotropy angle $\phi$. Solid line: ferromagnet, long-dashed line: antiferromagnet, dashed line: columnar antiferromagnet, dotted line: spiral. The vertical lines mark the phase boundaries, the special anisotropy angles for the antiferromagnetic square lattice $\left(J_{2}=0\right.$, $\square)$, the isotropic triangular antiferromagnet $\left(J_{2}=J_{1}, \triangle\right)$, and the antiferromagnetic chains $\left(J_{1}=0, \|\right)$ are indicated by the corresponding symbols above the plot. Bottom: Saturation field for triangular (solid line) and square lattice $J_{1}-J_{2}$ model (dashed line).
The classical energy then reads

$$
E_{\mathrm{cl}}=N S^{2}\left[J_{\perp}(\mathbf{Q})+A(0) \cos ^{2} \Theta-\frac{g \mu_{\mathrm{B}} \mu_{0} H}{S} \cos \Theta\right],
$$

where the coefficient $A(0)=A(\mathbf{q}=0)$ is defined by

$$
A(\mathbf{q})=J_{z}(\mathbf{q})+\frac{1}{2}\left[J_{\perp}(\mathbf{q}+\mathbf{Q})+J_{\perp}(\mathbf{q}-\mathbf{Q})\right]-2 J_{\perp}(\mathbf{Q}) .
$$

Minimizing Eq. (8) with respect to $\Theta$ yields the classical canting angle and saturation field $H_{\text {sat }}$ :

$$
\Theta_{\mathrm{c}}=\cos ^{-1}\left(\frac{H}{H_{\mathrm{sat}}}\right), \quad H_{\mathrm{sat}}=\frac{2 S A(0)}{\mu_{0} g \mu_{\mathrm{B}}},
$$

and we get

$$
E_{\mathrm{cl}}=N S^{2}\left[J_{\perp}(\mathbf{Q})-A(0)\left(\frac{H}{H_{\mathrm{sat}}}\right)^{2}\right] .
$$

The vector $\mathbf{Q}$ can be found by minimizing the above expression with respect to it. Setting $\partial E_{\mathrm{cl}} / \partial Q_{\alpha}=0, \alpha=x, y$ we find different types of solutions which are tabulated in Table $\mathrm{I}$, together with their classical energies. The last two columns of the table list the conditions and ranges of anisotropy angle where the corresponding solution $\mathbf{Q}$ describes the ground state.

Fig. 2illustrates the moment patterns of the different classical phases. In the isotropic case, the columnar antiferromagnet and the antiferromagnet are identical, because the former can be mapped onto the latter through a rotation of the lattice by an angle $2 \pi / 3$. Therefore their energy curves cross at $\phi=\pi / 4$ (Fig 3 (top)). However the ordering vector corresponding to the columnar antiferromagnet never minimizes the classical energy, and for $J_{1}=J_{2}>0(\phi=\pi / 4)$, the 120degree-structure characterizes the ground state (last row in Table I). Figure 3 (top) displays the dependence of the classical 


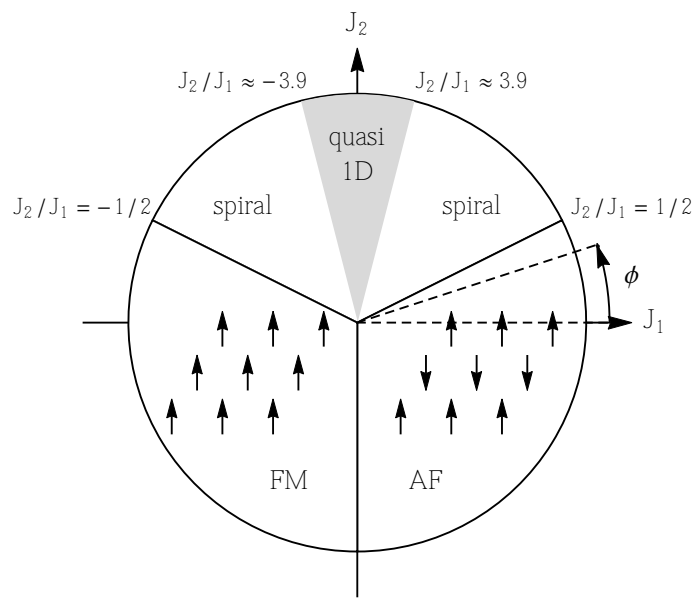

FIG. 4. Classical phase diagram. The phase boundaries are determined from the classical ground-state energy. The boundaries of the spiral phase are given by $J_{2} / J_{1}= \pm 1 / 2$, the boundary between AF and $\mathrm{FM}$ is given by $J_{1}=0, J_{2} \geq 0$. The shaded area bounded by $J_{2} / J_{1} \approx \pm 3.9(0.42 \pi \leq \phi \leq 0.58 \pi)$ denotes the region where quantum fluctuations destroy the ordered moment within our semiclassical approximation. This area is centered around $J_{1}=0$, which corresponds to one-dimensional chains. The dashed lines illustrate the definition of the anisotropy angle $\phi$ with $-\pi \leq \phi \leq \pi$.

energies on the anisotropy angle $\phi$ while Figure 3 (bottom) shows the saturation fields in comparison of triangular and square lattice $J_{1}-J_{2}$ model.

The phase diagram according to this analysis is composed of three phases, a uniform ferromagnet (FM), a Néel antiferromagnet (AF), and a spiral phase with an in general incommensurate ordering vector, see Fig. 4. The columnar antiferromagnet has an energy which is never the lowest, therefore it is not realized as a ground state.

One set of ordering vectors has the form $\mathbf{Q}=( \pm Q, 0)$ for all three classical phases, shown in Fig. 5. In the spiral phase, $Q$ continuously interpolates between the antiferromagnet $(Q=2 \pi)$ and the ferromagnet $(Q=0)$. In this phase, $Q(\phi)=2 \pi-Q(\pi-\phi)$ is antisymmetric with respect to the point $(\phi, Q)=(\pi / 2, \pi)$ (antiferromagnetic chain with $\left.J_{1}=0, J_{2}>0\right)$ and has an infinite slope at the boundaries. The isotropic triangular lattice with $J_{1}=J_{2}$ is characterized by $Q(\pi / 2)=4 \pi / 3$ leading to the $120^{\circ}$ spin structure.

The phase diagram has a corresponding mirror symmetry with respect to the line $J_{1}=0$ : We may split the lattice into a sublattice $A$ having only sites with a particular spin direction, e.g. "up", in the AF phase, and a sublattice $B$ with sites all having a "down" spin. If all $B$ spins are flipped the Hamiltonian (1) with $\mathbf{H}=0$ remains invariant provided we simultaneously set $J_{1} \rightarrow-J_{1}$. This operation exchanges the FM and AF phases and leaves the spiral phase invariant. Exactly at $J_{1}=0$, the lattice is decoupled into independent chains, and one can rotate all spins on any chain by an arbitrary angle without energy cost.

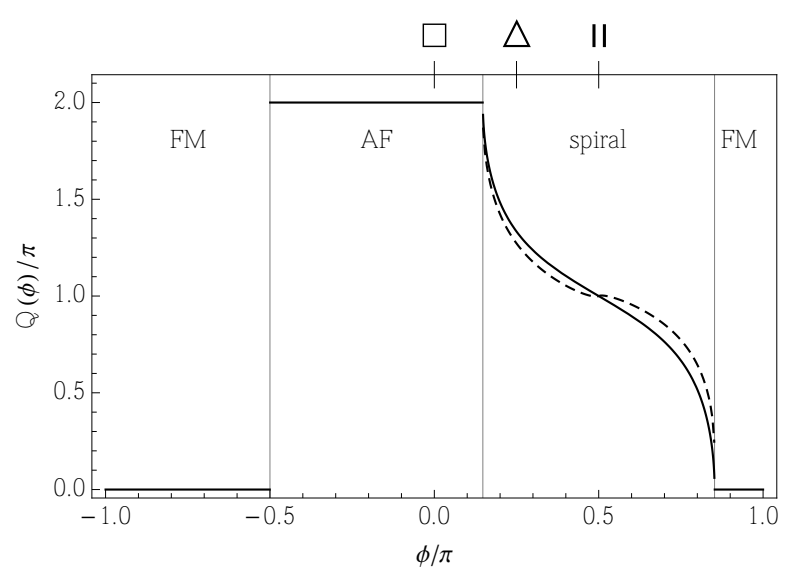

FIG. 5. Dependence of the component $Q$ of the ordering vector $\mathbf{Q}=( \pm Q, 0)$ on the anisotropy angle $\phi$. The solid line denotes the classical behavior, the dashed line denotes the $\phi$-dependence including first-order quantum corrections.

\section{QUANTUM FLUCTUATIONS}

The classical picture of magnetic order in the anisotropic trigonal model discussed sofar serves as a starting point to the main topic of this work: A global understanding of the influence of quantum fluctuations on the type, energy, ordered moment and magnetization of the ground state as function of the anisotropy angle $\phi$. For that purpose we employ a standard linear spin wave analysis of magnetic excitations and calculate the effect of zero point fluctuations on these quantities. Naturally, as in the square lattice model, regions of $\phi$ where the moment becomes unstable and spin wave approximation breaks down will appear. It is important to characterize the size of those regions and get clues on the type of nonmagnetic state that may appear.

To perform the calculation of quantum corrections we return to the Hamiltonian (1) expressed in the local coordinate system introduced in the previous section, we apply a Holstein-Primakoff transformation and carry out a large- $S$ expansion, keeping terms up to fist order in $1 / S$. We regard the magnetic field $\mathbf{H}$ formally as proportional to $S$. Applying a Fourier transformation and a subsequent Bogoliubov transformation leads in the thermodynamic limit to the familiar general form

$$
\mathscr{H}=E_{\mathrm{cl}}+E_{\mathrm{zp}}+N S \int \frac{\mathrm{d}^{2} q}{V_{\mathrm{BZ}}} \omega_{\mathrm{sw}}(\mathbf{q}) \alpha_{\mathbf{q}}^{\dagger} \alpha_{\mathbf{q}}
$$

where the $q$ integration is to be taken over the first Brillouin zone (BZ) with area $V_{\mathrm{BZ}}=2 \sqrt{3} \pi^{2}, E_{\mathrm{cl}}$ is given by Eq. 8 , and $\alpha_{\mathbf{q}}^{\dagger}$ denotes a magnon with wave vector $\mathbf{q}$. The zero-point energy $E_{\mathrm{zp}}$ and magnon dispersion $\omega_{\mathrm{sw}}$ are given by

$$
\begin{aligned}
E_{\mathrm{zp}} & =N S\left(J_{\perp}(\mathbf{Q})+\frac{1}{2} \int \frac{\mathrm{d}^{2} q}{V_{\mathrm{BZ}}} \omega(\mathbf{q})\right), \\
\omega_{\mathrm{sw}}(\mathbf{q}) & =\omega(\mathbf{q})+C(\mathbf{q}) \frac{H}{H_{\mathrm{sat}}},
\end{aligned}
$$



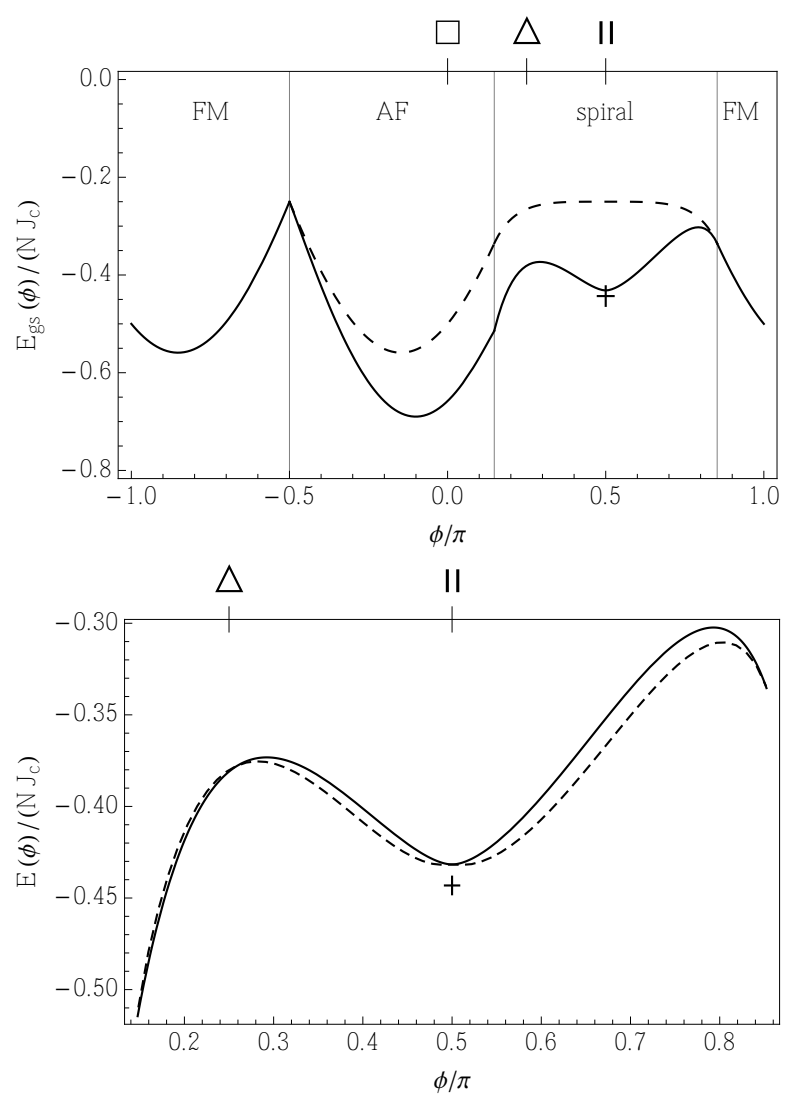

FIG. 6. Ground-state energy $E_{\mathrm{gs}}$ for $S=1 / 2$ including first-order quantum corrections as a function of $\phi$ (solid line). Cross at $\phi=\pi / 2$ : Exact $1 D$ Bethe ansatz result $E_{\mathrm{gs}} /\left(N J_{\mathrm{c}}\right)=1 / 4-\ln 2$. Top: Full range of anisotropy angles. Dashed line: Classical ground-state energy. Bottom: Zoom into the spiral phase. Dashed line: small corrections to $E_{\mathrm{gs}}$ in the spiral phase due to ordering vector corrections.

$$
\begin{aligned}
\omega(\mathbf{q})= & \left\{\left[A(\mathbf{q})-B(\mathbf{q})\left(H / H_{\text {sat }}\right)^{2}\right]^{2}\right. \\
& \left.-\left[B(\mathbf{q})\left(1-\left(H / H_{\text {sat }}\right)^{2}\right)\right]^{2}\right\}^{1 / 2} .
\end{aligned}
$$

The coefficients $B$ and $C$, the latter only being present at finite magnetic fields $\mathbf{H}$, are given by

$$
\begin{aligned}
& B(\mathbf{q})=J_{z}(\mathbf{q})-\frac{1}{2}\left[J_{\perp}(\mathbf{q}+\mathbf{Q})+J_{\perp}(\mathbf{q}-\mathbf{Q})\right], \\
& C(\mathbf{q})=J_{\perp}(\mathbf{q}+\mathbf{Q})-J_{\perp}(\mathbf{q}-\mathbf{Q}) .
\end{aligned}
$$

We note that $C(\mathbf{q})$ is nonzero only in the spiral phase and does not contribute to the ground-state energy $E_{\mathrm{gs}}=E_{\mathrm{cl}}+E_{\mathrm{zp}}$.

For the determination of the ordering vector $\mathbf{Q}$, we now have to minimize the full ground-state energy $E_{\mathrm{gs}}=E_{\mathrm{cl}}+E_{\mathrm{zp}}$ as given by Eqs. (8) and (13), again up to first order in $1 / S$. For $\mathbf{H}=0$, this amounts to finding the roots of the equation

$$
-\frac{\partial J_{\perp}(\mathbf{Q})}{\partial Q_{\alpha}}=\left.\frac{1}{2 S} \int \frac{\mathrm{d}^{2} q}{V_{\mathrm{BZ}}}\left(\frac{\partial A(\mathbf{q})}{\partial Q_{\alpha}} \cdot \frac{A(\mathbf{q})+B(\mathbf{q})}{\omega(\mathbf{q})}\right)\right|_{\mathbf{Q}=\mathbf{Q}_{\mathrm{cl}}}
$$
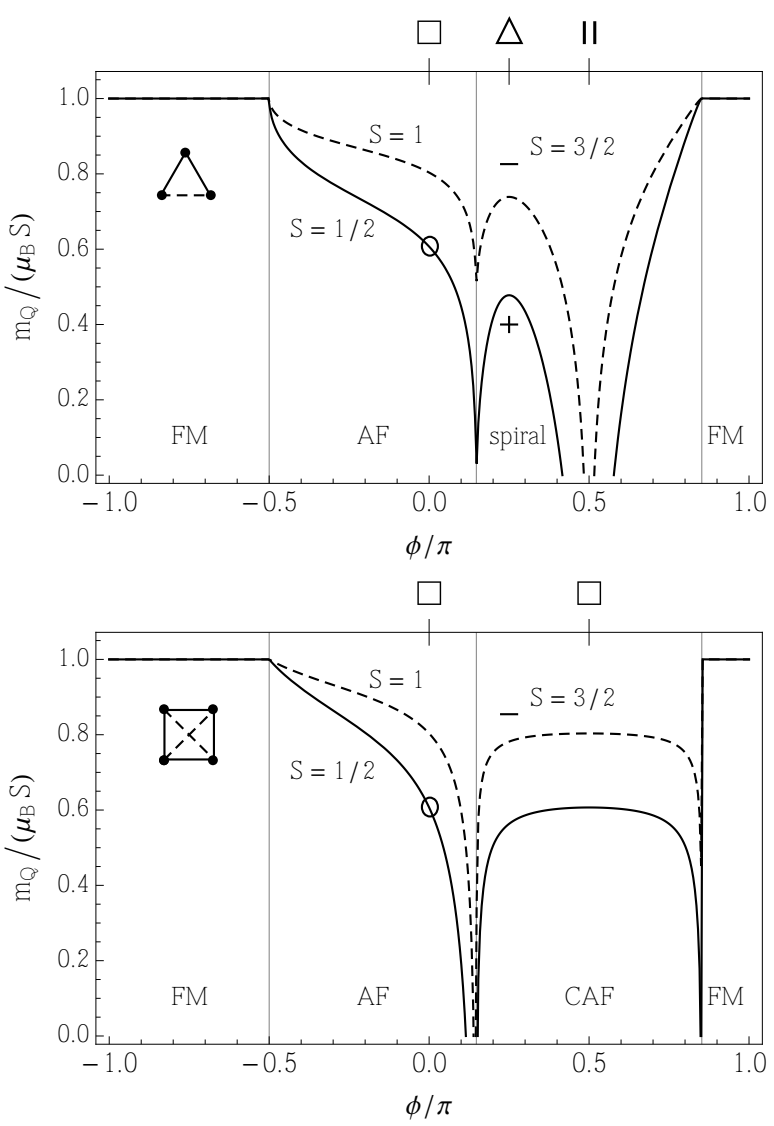

FIG. 7. Size of the ordered moment $m_{Q}$ according to Eq. (19) as a function of the anisotropy angle $\phi$. Top: anisotropic triangular lattice. Bottom: $J_{1}-J_{2}$ model on the square lattice. The solid lines denote $S=1 / 2$, the circle marks the ordered moment for the square-lattice antiferromagnet. The cross marks the DMRG result $m_{Q} /\left(\mu_{\mathrm{B}} S\right)=0.41$ [5]. The dotted lines show the $\phi$ dependence of $m_{Q}$ for $S=1$, and the bar at $\phi=\pi / 4$ denotes $m_{Q}$ for $S=3 / 2$.

for the components of $\mathbf{Q}$. In the AF and FM phases, the righthand side of this equation vanishes, and Eq. (18) leads again to the classical values a given in Table 1 . Small but finite corrections to these appear in the spiral phase only, see the dashed line of Fig.5. Unless explicitly noted, we ignore these as well as spin-space anisotropies $\left(J_{i j}^{\perp}=J_{i j}^{z}\right)$ in the following.

\section{GROUND-STATE ENERGY}

In the ferromagnetic phase, corrections to the ground-state energy are absent. Quantum fluctuations strongly renormalize the ground-state energy $E_{\mathrm{gs}}$ in the antiferromagnetic and spiral phases. The solid line in Fig. 6 shows a plot of $E_{\mathrm{gs}}$ for $S=1 / 2$ as introduced in the preceding section, calculated using the classical ordering vector. For comparison, the dashed line in the upper part of the figure displays the classical groundstate energy $E_{\mathrm{cl}}$. The reflection symmetry of the classical energy around the one-dimensional point $(\|)$ is destroyed by the quantum fluctuations. 
While vanishing at the boundaries to the ferromagnet, the quantum corrections are largest in the spiral phase, in particular around the one-dimensional point $\phi=\pi / 2\left(J_{1}=0\right)$. The lower part of Fig. 6 gives a zoom into this phase and shows the additional small corrections due to the ordering vector corrections introduced in the preceding section. The cross at $\phi=\pi / 2$ denotes the exact Bethe ansatz result $E_{\mathrm{gs}} /\left(N J_{\mathrm{c}}\right)=1 / 4-\ln 2 \approx-0.443$ for the one-dimensional AF chain [29, [30]. From linear spin-wave theory we get $E_{\mathrm{gs}} /\left(N J_{\mathrm{c}}\right)=-3 / 4+1 / \pi \approx-0.432$.

\section{ORDERED MOMENT}

Quantum fluctuations reduce the ordered moment $m_{Q}$ from its classical value $m_{Q}^{\mathrm{cl}}=\mu_{\mathrm{B}} S$. Including their effect it has the general form

$$
m_{Q}=\mu_{\mathrm{B}} S\left[1-\frac{1}{2 S}\left(\int \frac{\mathrm{d}^{2} q}{V_{\mathrm{BZ}}} \frac{A(\mathbf{q})}{\omega(\mathbf{q})}-1\right)\right]
$$

for field $\mathbf{H}=0$. Fig. 7 shows a plot of the ordered moment $m_{Q}$ at field $\mathbf{H}=0$ as a function of the anisotropy angle $\phi$. We note that the absolute correction to the classical value $m_{Q}^{\mathrm{cl}}$ does not depend on $S$. In the FM phase, the classical value remains unchanged. In the AF phase, quantum fluctuations reduce the ordered moment smoothly form a value $m_{Q}=\mu_{\mathrm{B}} S$ in the FM phase to $m_{Q}=0$ at $J_{2} / J_{1}=1 / 2(\phi \approx 0.148 \pi)$. At $\phi=0$, we get the well-known value $m_{Q} /\left(\mu_{\mathrm{B}} S\right) \approx 0.606$ for $S=1 / 2$ (small circle in Fig. 7).

In the spiral phase for $\phi \geq \tan ^{-1}(1 / 2)$, the ordered moment is stabilized until the commensurate $120^{\circ}$-spiral state with ordering vector component $Q=4 \pi / 3$ is reached at $\phi=\pi / 4$ (isotropic case), and $m_{Q} /\left(\mu_{\mathrm{B}} S\right) \approx 0.478$ for $S=1 / 2$ [2]. Increasing the ratio $J_{2} / J_{1}$ further, the ordered moment is suppressed until it vanishes at $\phi / \pi \approx 0.42$, corresponding to $J_{2} / J_{1} \approx 3.9$. For $\phi / \pi>0.58$ or $J_{2} / J_{1}>-3.9$, the ordered moment is stabilized and monotonously increases until it reaches the border to the FM phase at $J_{2} / J_{1}=-1 / 2$ $(\phi / \pi \approx 0.852)$, where the classical value is restored. The region with $J_{2} /\left|J_{1}\right|>3.9$ indicates a classically not present disordered regime around the point $\phi=\pi / 2$, where $J_{1}=0$. This point denotes the one-dimensional antiferromagnetic chain which has no long range magnetic order but only quasi-long range algebraic spin correlations [31].

Turning on a magnetic field, the moments on each site cant towards the field direction defining our global $z$ axis. The ordered moment which is the projection of the former onto the plane perpendicular to $z$ can then be written as [32]

$$
m_{Q}(H)=\mu_{\mathrm{B}} S \sqrt{1-\left(\frac{H}{H_{\mathrm{sat}}}\right)^{2}}\left\{1-\frac{1}{1-\left(H / H_{\mathrm{sat}}\right)^{2}} \frac{1}{2 S} \int \frac{\mathrm{d}^{2} q}{V_{\mathrm{BZ}}}\left[\frac{A(\mathbf{q})}{\omega(\mathbf{q})}-1+\left(\frac{H}{H_{\mathrm{sat}}}\right)^{2} \frac{B(\mathbf{q})}{\omega(\mathbf{q})}\left(\frac{A(\mathbf{q})-B(\mathbf{q})}{A(0)}-1\right)\right]\right\}
$$

up to $\mathscr{O}(1 / S)$, including corrections to the classical canting angle $\Theta_{c}$. Figure 8 displays the field dependence of the ordered moment for $S=1 / 2$ and different anisotropy angles $\phi$. For comparison also the classical $S \rightarrow \infty$ field dependence is shown (topmost curve). In Fig. 7 for all four values of $\phi$ the ordered moment at $H=0$ is reduced from its classical value by quantum fluctuations. We have chosen characteristic values for the anisotropy: $\phi=0.4 \pi$ (lowest solid line) is near the crossover to the quasi-onedimensional behavior. The dashed curve has $\phi=\pi / 4$, which is the isotropic triangular antiferromagnet starting at $m_{Q}(H=0) /\left(\mu_{B} S\right)=0.478$. The dotted curve with $\phi / \pi=0.18$ is at the AF/spiral boundary. The zero-field moments for $\phi / \pi=0.4,0.18$ are both lower than for the isotropic trigonal case $\phi=0.25 \pi$. This means that in the corresponding spiral phase region $m_{Q}(H=0, \phi) /\left(\mu_{B} S\right)$ is non-monotonic as function of control parameter $\phi$. Finally the dash-dotted curve for $\phi=0$ corresponds to the unfrustrated square-lattice antiferromagnet. The moment reduction is strongest near the quasi-onedimensional region. Upon increasing $H$, two effects determine $m_{Q}(H)$ : (a) Due to the suppression of quantum fluctuations by the magnetic field, the total moment is stabilized, leading to an initial increase of $m_{Q}(H)$. (b) The total moment is tilted towards the direction of the magnetic field, leading to a decrease of $m_{Q}(H)$, which at sufficiently large fields dominates the field dependence of
$m_{Q}(H)$. In the classical limit, $m_{Q}(H)$ is determined only by the tilting angle $\Theta_{c}$ relative to the field direction. The initial slope $\lim _{H \rightarrow 0} m_{Q} / H$ therefore can be used as a means to determine the anisotropy angle $\phi$ from neutron diffraction experiments in a magnetic field as has already been demonstrated for the square-lattice $J_{1}-J_{2}$ model [32].

\section{MAGNETIZATION AND SUSCEPTIBILITY}

The magnetization $M$ can be derived from the ground-state expectation value of the $z$ component of the total spin $\mathbf{S}=$ $\sum_{i} \mathbf{S}_{i}$. It is given by

$$
M=\frac{N}{V} \frac{\left(g \mu_{\mathrm{B}}\right)^{2} \mu_{0} H}{2 A(0)}\left[1+\frac{1}{2 S} \int \frac{\mathrm{d}^{2} q}{V_{\mathrm{BZ}}} \frac{B(\mathbf{q})(A(\mathbf{q})-B(\mathbf{q}))}{A(0) \omega(\mathbf{q})}\right]
$$

where $V$ denotes the sample volume. We note that $\omega(\mathbf{q})$ also depends on $H$, see Eq. (15). For $S=1 / 2$, Fig. 9 displays a plot of $M(H)$ for different values of the anisotropy angle $\phi$, together with the classical result. Both field $H$ and magnetization $M$ are normalized to their saturation values $H_{\text {sat }}$ and $M_{\mathrm{sat}}=(N / V) g \mu_{\mathrm{B}} S$, respectively. For better distinguishability, the individual curves are offset by 0.3 . Deep within the antiferromagnetic phase (example: $\phi=-\pi / 4$ ), the corrections to 

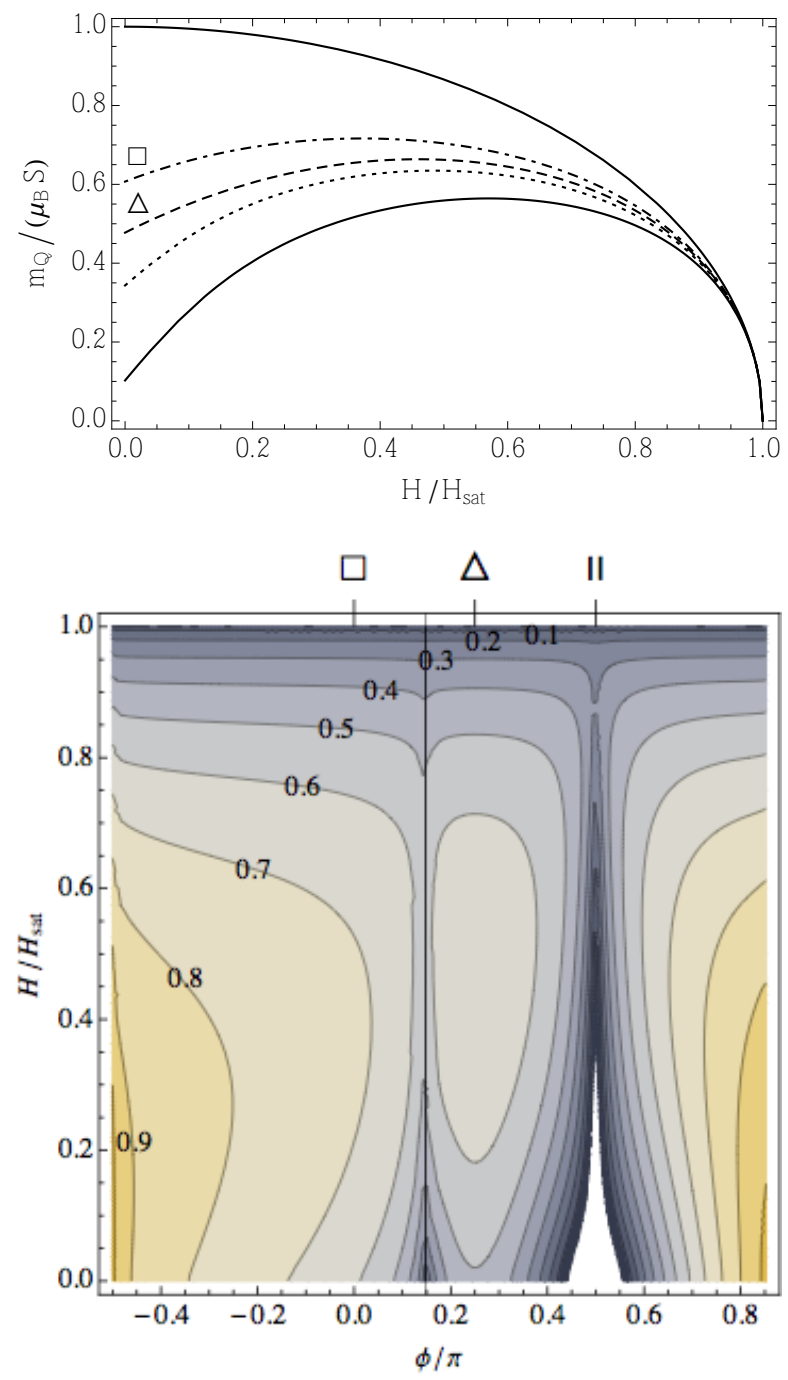

FIG. 8. Top: Field dependence of the ordered moment $m_{Q}(H)$ for $S=1 / 2$ at different anisotropy angles $\phi$. Lowest solid line: $\phi=0.4 \pi-$ near the crossover to quasi-onedimensionality, dashed line: $\phi=\pi / 4$ - isotropic triangular antiferromagnet, dotted line: $\phi=0.18 \pi-$ near the boundary between spiral and antiferromagnetic phase, dot-dashed line: $\phi=0$ - square-lattice antiferromagnet. The upper (solid) line describes the classical $S \rightarrow \infty$ behavior. Bottom: $\phi-H$ contour plot of the ordered moment. Instability regions around $\phi / \pi=\tan ^{-1}(1 / 2) / \pi \approx 0.15$ and 0.5 become narrower for increasing $\mathrm{H}$. Labels denote $0 \leq m_{Q} /\left(\mu_{B} S\right) \leq 1$ in steps of 0.1 .

the classical field dependence are small, and increase with increasing $\phi$. At $\phi=0$, the field dependence corresponds to the square-lattice antiferromagnet. At $\phi=0.4 \pi$ or $J_{2} / J_{1} \approx 3.1$, the border of the stability range of the ordered moment is reached, and corrections to $M(H)$ lead to a strong curvature in particular at small fields. For even larger values of $\phi$, the fluctuations overcompensate the classical value for $M$, leading to an unphysical negative magnetization at small fields. As an example, we show $M(H)$ for $\phi=3 \pi / 4$, where the dashed line indicates this overcompensation. We also note that the magnetization plateau at $M / M_{\text {sat }}=1 / 3$ [33] cannot be obtained within LSW since it is due to bound state formation of spin

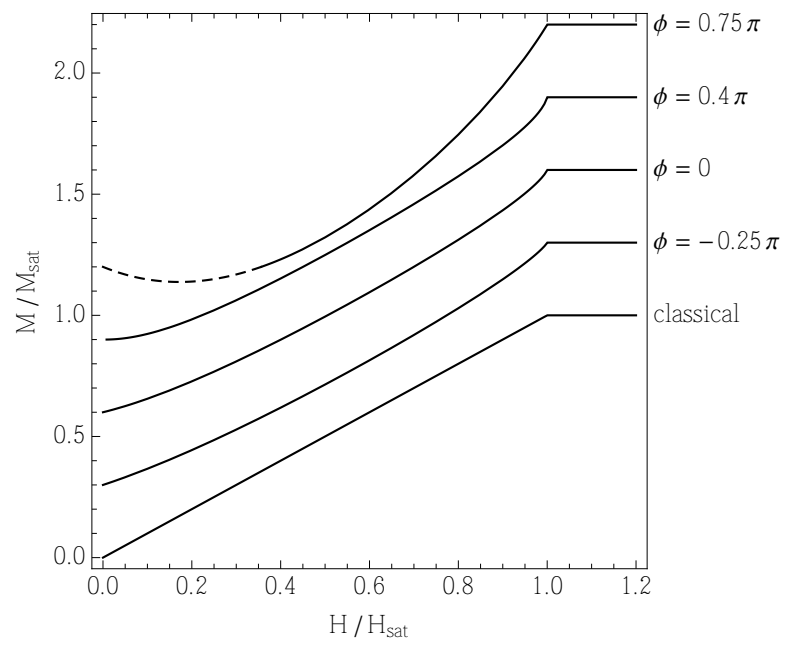

FIG. 9. Homogeneous magnetization $M$ for $S=1 / 2$ in units of the saturation magnetization $M_{\text {sat }}=(N / V) g \mu_{\mathrm{B}} S$. Shown are curves for the classical limit and for different values of the anisotropy angle with an offset of 0.3 . The dashed line for $\phi=0.75 \pi$ denotes the range where fluctuations overcompensate the classical value. The magnetic field is normalized to the (anisotropy-dependent) saturation value $H_{\text {sat }}=2 S A(0) /\left(\mu_{0} g \mu_{\mathrm{B}}\right)$ (Fig. 3 ).

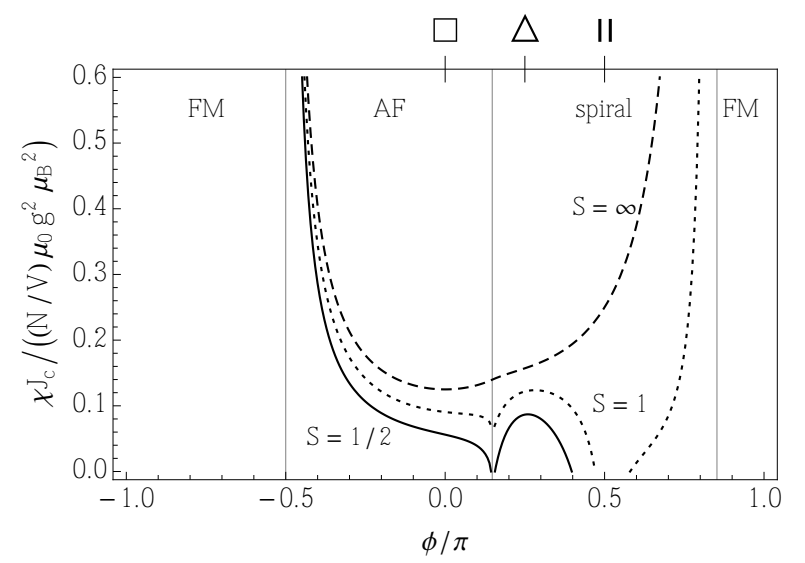

FIG. 10. Magnetic susceptibility $\chi=\lim _{H \rightarrow 0} M / H$ as a function of the anisotropy angle $\phi$. Solid line: $S=1 / 2$, dotted line: $S=1$, dashed line: classical limit $S \rightarrow \infty$.

waves. To illustrate the small-field corrections to the classical case more clearly, Fig. 10 shows a plot of the magnetic susceptibility $\chi=\lim _{H \rightarrow 0} M / H$ as a function of the anisotropy angle $\phi$. The solid line denotes the anisotropy dependence for $S=1 / 2$, the dotted line shows $S=1$, and the dashed line shows the field dependence for $S \rightarrow \infty$. Already for $S=1$, the unphysical range of $\phi$ is restricted to values around the one-dimensional case $\phi=\pi / 2$.

\section{EXCITATION SPECTRA}

Here we discuss the systematic variation of the LSW excitation spectrum when the control parameter $\phi$ is tuned through 

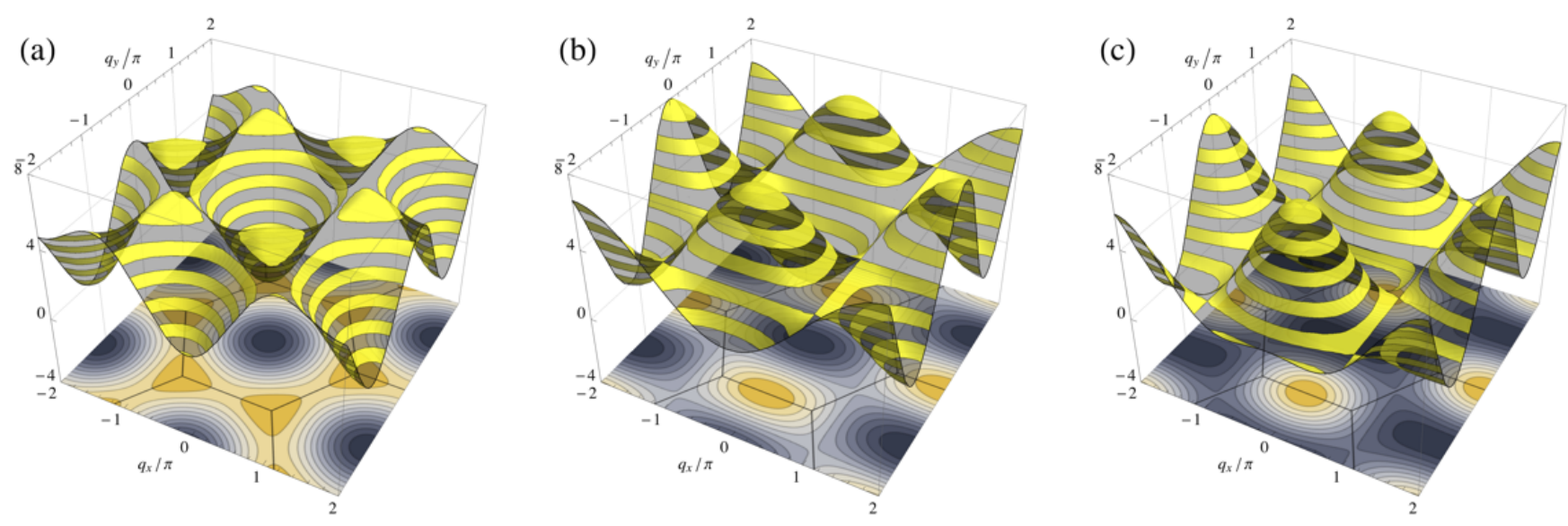

FIG. 11. Ferromagnetic spin wave dispersion $\omega_{\mathrm{sw}}(\mathbf{q})$ in units of $J_{\mathrm{c}}$. From left to right: (a) $J_{1}=J_{2}-$ isotropic triangular ferromagnet, (b) $J_{2}=0$ - square-lattice ferromagnet, (c) $J_{1} \approx-2 J_{2}, J_{2}>0$ - border with spiral phase.

the phase diagram of Fig. 4. We also emphasize the variation of anisotropic spin wave velocities, their measurement may be useful for determination of $\phi$.

Ferromagnet. The fully polarized state remains the unchanged ground state of the Hamiltonian for $J_{1} \leq 0$ and $J_{2} /\left|J_{1}\right| \leq 1 / 2$, however its excitation spectrum strongly depends on the anisotropy parameter $\phi$. It is given by

$$
\begin{aligned}
\omega_{\mathrm{sw}}(\mathbf{q})= & -4\left(J_{1}+\frac{1}{2} J_{2}\right) \\
& +4\left(J_{1} \cos \frac{q_{x}}{2} \cos \frac{\sqrt{3}}{2} q_{y}+\frac{1}{2} J_{2} \cos q_{x}\right) .
\end{aligned}
$$

The dispersion for selected values of the anisotropy parameter $\phi$ is shown in Fig. 11. Crossing the border from the Néel antiferromagnet, $J_{1}$ changes sign, and the dispersion is identical to that of a one-dimensional ferromagnetic chain (quadratic dispersion along $q_{x}$ at $\mathbf{q}=0$ ). Deep within the ferromagnetic region, the long-wavelength dispersion around the ordering vector $\mathbf{Q}=0$ can be written as

$$
\omega_{\mathrm{sw}}(\mathbf{q}) \approx \frac{D_{x}}{S} q_{x}^{2}+\frac{D_{y}}{S} q_{y}^{2}
$$

introducing two stiffness constants $D_{\alpha}$ given by

$$
D_{x}=-\frac{S}{2}\left(J_{1}+2 J_{2}\right), \quad D_{y}=-\frac{3}{2} S J_{1},
$$

which are positive and identical in the isotropic case $J_{1}=J_{2}$. The full spectrum for this case is shown in Fig. 11 a, having a sixfold symmetry around the zeroes at the Bragg points $\mathbf{Q}^{*}=$ $2 \pi(n, m / \sqrt{3})$ with $n, m \in \mathbb{Z}$. Figure $11 \mathrm{p}$ shows the case $J_{2}=$ 0 , which is a pure square-lattice ferromagnet with exchange constant $J_{1}$. Apart from a scaling factor $1 / \sqrt{3}$ for $q_{y}$, this is reflected in the fourfold-symmetric shape of the spectrum around the Bragg points. At the border to the spiral phase for $J_{2}=\left|J_{1}\right| / 2$ shown in plot (c), $D_{x}$ vanishes, indicating the instability towards incommensurate order.
Antiferromagnet. Fig. 12 displays a series of plots for the spin wave dispersion in the two-sublattice antiferromagnetic phase. It is given by

$$
\begin{aligned}
\omega_{\mathrm{sw}}(\mathbf{q}) & =\sqrt{A^{2}(\mathbf{q})-B^{2}(\mathbf{q})}, \\
A(\mathbf{q}) & =4\left(J_{1}-\frac{1}{2} J_{2}\right)+2 J_{2} \cos q_{x}, \\
B(\mathbf{q}) & =4 J_{1} \cos \frac{q_{x}}{2} \cos \frac{\sqrt{3}}{2} q_{y} .
\end{aligned}
$$

As expected, the intra-sublattice dispersion $A(\mathbf{q})$ only depends on $q_{x}$. An expansion around the ordering vector $\mathbf{Q}$ yields an expression

$$
\omega_{\mathrm{sw}}^{2}(\mathbf{q}) \approx\left(\frac{\hbar v_{x}}{S \ell_{x}}\right)^{2}\left(q_{x}-Q_{x}\right)^{2}+\left(\frac{\hbar v_{y}}{S \ell_{y}}\right)^{2}\left(q_{y}-Q_{y}\right)^{2}
$$

where $\ell_{x, y}$ denote the lattice spacings along $x$ and $y$ directions. $\left(\ell_{x}=a, \ell_{y}=(\sqrt{3} / 2) a\right.$ for the geometrically isotropic triangular lattice with lattice constant $a$.) The anisotropic spin-wave velocities are given by

$$
v_{x}=\frac{S \ell_{x}}{\hbar} 2 J_{1} \sqrt{1-2 \frac{J_{2}}{J_{1}}}, \quad v_{y}=\frac{S \ell_{y}}{\hbar} 2 \sqrt{3} J_{1} .
$$

Fig. 13 shows the evolution of $v_{x, y}$ as a function of the anisotropy angle $\phi$. At the border to the FM phase, $v_{x, y}$ increase from $v_{x}=v_{y}=0$ and the change from quadratic q dependence from Eq. 23] to the low-energy linear dispersion given by Eq. 28) around the points $\mathbf{Q}^{*}$ is observed. Towards the inside of the AF phase, these dispersion cones stabilize and, for small excitation energies, eventually get nearly isotropic for $J_{2}=-J_{1}$ or $\phi=-\pi / 4$ (Fig. 12p) with $v_{x} / \ell_{x}=$ $v_{y} / \ell_{y}=2 \sqrt{3} S J_{1} / \hbar$. For high energies however, the flat $q_{y}$ dependence of $\omega_{\mathrm{sw}}(\mathbf{q})$ remains. For $J_{2}=0$, the model describes the familiar square-lattice Néel antiferromagnet with exchange coupling $J_{1}$, see Fig. 12p. Towards the spiral phase, new maxima in $\omega_{\mathrm{sw}}(\mathbf{q})$ develop. The low-energy cones become soft in $q_{x}$ direction until at $J_{2}=J_{1} / 2$, the spin-wave 

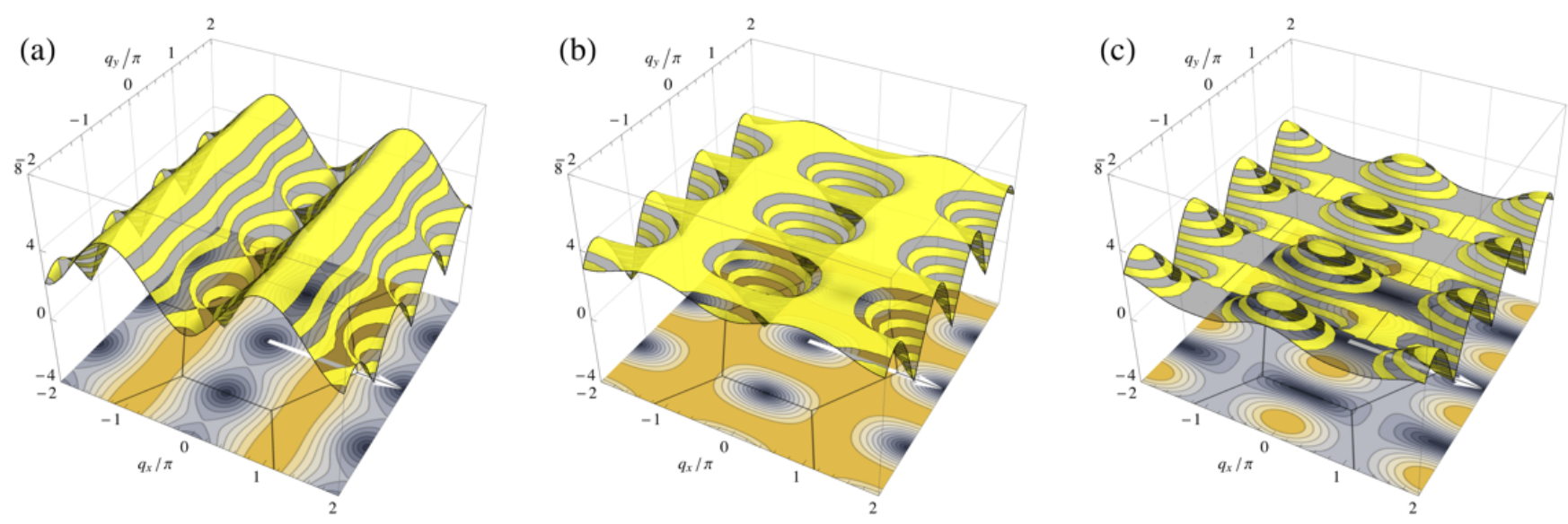

FIG. 12. Antiferromagnetic spin wave dispersion $\omega_{\mathrm{sw}}(\mathbf{q})$ in units of $J_{\mathrm{c}}$. From left to right: (a) $J_{1}=\left|J_{2}\right|-$ deep inside antiferromagnetic phase, (b) $J_{2}=0$ - square-lattice antiferromagnet, (c) $J_{1} \approx 2 J_{2}>0$ - border with spiral phase. The white arrow denotes the ordering vector $\mathbf{Q}=(2 \pi, 0)$.

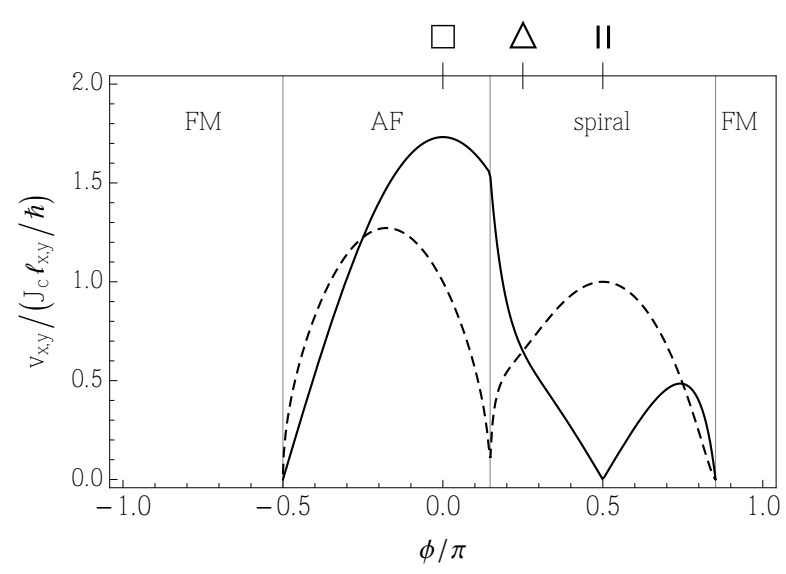

FIG. 13. Evolution of the spin-wave velocities $v_{x}, v_{y}$ in the antiferromagnetic and spiral phases as a function of the anisotropy angle $\phi$. Dashed line: $v_{x}$, solid line: $v_{y}$.

velocity $v_{x}$ vanishes (Fig. 13). Note that this does not lead to line zeros in the spectrum. Minima in the antiferromagnetic phase occur both in the center of the chemical Brillouin zone and at the border of it with $q_{y}=n \cdot 2 \pi / \sqrt{3}, n \in \mathbb{Z}$.

Spiral phase. Between $\phi=\tan ^{-1}(1 / 2)$ and $\phi=\pi-$ $\tan ^{-1}(1 / 2)$, the ordering vector in general is incommensurate and has the form $\mathbf{Q}=\left(Q_{x}, 0\right)$ with $0 \leq Q_{x} \leq 2 \pi$. The spinwave spectra obtained are plotted in Fig. 14 The dispersion is again given by Eq. 25$)$, and the coefficients $A(\mathbf{q})$ and $B(\mathbf{q})$ retain the general forms given by Eqs. (9) and (16). Expanding $\omega_{\mathrm{sw}}(\mathbf{q})$ around the ordering vector leads to a linear $\mathbf{q}$ dependence around the dispersion minima given by Eq. (28). The spin-wave velocities in this case are

$$
v_{x}=\frac{S \ell_{x}}{\hbar} 2 J_{2}\left(1+\frac{J_{1}}{2 J_{2}}\right)^{3 / 2}
$$

$$
\begin{gathered}
\times \sqrt{1-\frac{3}{2} \frac{J_{1}}{J_{2}}+\left(\frac{J_{1}}{J_{2}}\right)^{2}-\frac{1}{4}\left(\frac{J_{1}}{J_{2}}\right)^{3}}, \\
v_{y}=\frac{S \ell_{y}}{\hbar} \sqrt{3} J_{1}\left(1+\frac{J_{1}}{2 J_{2}}\right) \sqrt{1-\frac{J_{1}}{J_{2}}+\frac{1}{2}\left(\frac{J_{1}}{J_{2}}\right)^{2}} .
\end{gathered}
$$

In the isotropic case $\left(J_{1}=J_{2}=J_{\mathrm{c}} / \sqrt{2}\right)$, we have $\hbar v_{x} / \ell_{x}=$ $\hbar v_{y} / \ell_{y}=(3 \sqrt{3} / 4) S J_{\mathrm{c}} \approx 1.3 S J_{\mathrm{c}}$ (Fig. 13). Increasing $\phi$ from the border to the antiferromagnetic phase, we have a crossover from the fourfold symmetry of the Néel antiferromagnet to the sixfold symmetry of the isotropic triangular magnet, see Fig. 14a. Low-energy cones with linear q dependence develop in the center and at the corners of the Brillouin zone. Upon further increase of $\phi$, the sixfold symmetry is transformed into the twofold-symmetric one-dimensional pattern, see Fig. 14b. For ferromagnetic $J_{1}<0$ (but still antiferromagnetic $J_{2}$ and an in general incommensurate ordering vector), the excitation spectrum becomes similar to what we have found in the ferromagnetic phase.

\section{DISCUSSION}

We begin the further discussion of our results with the classical phase diagram. It results from the classical ground state energies plotted in Fig. 3. The phase boundaries appear at the same values of $\phi$ as in the case of the square lattice $J_{1}-J_{2}$ model. However in the range $0.15 \leq \phi / \pi \leq 0.85$ the ground state for the triangular model is a generally incommensurate (IC) spiral whereas the square lattice model has a commensurate (C) columnar AF ground state. The reason may be understood from Fig. 3. In the above range the lowest (dotted) energy curve of the spiral phase is only slightly lower than the one for the columnar AF (short-dashed) which shows a simple $-J_{c} \sin \phi=-J_{2}$ dependence. They touch at $\phi / \pi=0.5$ for the decoupled chain model. In the case of the square lattice model, however, there is one diagonal $J_{2}$ exchange bond more, 

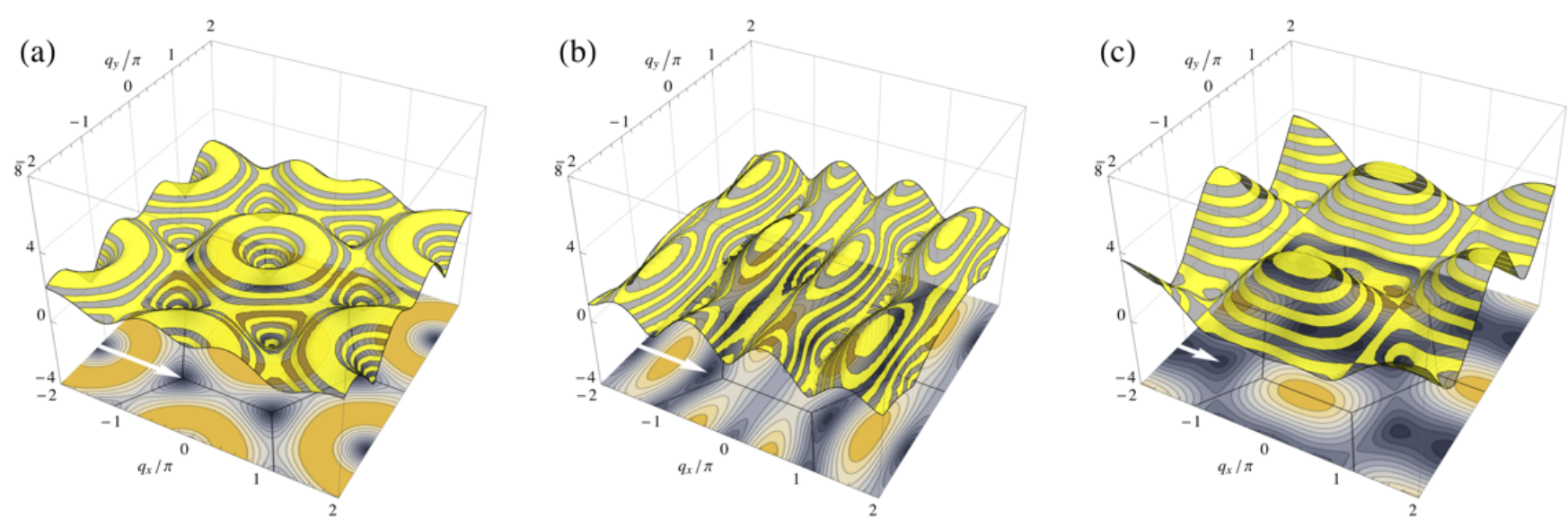

FIG. 14. Spin wave dispersion $\omega_{\mathrm{sw}}(\mathbf{q})$ in units of $J_{\mathrm{c}}$ in the spiral phase. From left to right: (a) $J_{2}=J_{1}$ - isotropic triangular antiferromagnet, (b) $J_{2} / J_{1} \approx 3$ - border to disordered region, (c) $J_{2}=-J_{1}>0$ - spiral with ferromagnetic $J_{1}$. For better visibility, the white arrows showing the ordering vector $\mathbf{Q}$ are shifted by $\Delta \mathbf{Q}=-(2 \pi, 2 \pi / \sqrt{3})$. The case (b) corresponds approximately to $\mathrm{Cs}_{2} \mathrm{CuCl}_{4}\left(\mathrm{see} \mathrm{Table}_{\mathrm{II}}\right.$ )

TABLE II. Exchange parameters of some compounds with frustrated triangular spin structure

\begin{tabular}{|c|c|c|c|c|c|c|c|c|}
\hline compound & $S$ & $J_{2} / J_{1}$ & $\phi / \pi$ & $J_{\mathrm{c}} / k_{\mathrm{B}}$ & $Q_{\mathrm{cl}} / \pi$ & $m_{Q}^{\mathrm{sw}} /\left(\mu_{\mathrm{B}} S\right)(\exp )$ & {$\left[\left(v_{x} / \ell_{x}\right) /\left(v_{y} / \ell_{y}\right)\right]_{\mathrm{sw}}$} & reference \\
\hline $\mathrm{Cs}_{2} \mathrm{CuCl}_{4}$ & $1 / 2$ & 3.31 & 0.41 & $4.9 \mathrm{~K}$ & 1.10 & 0.043 & 3.93 & 34 \\
\hline $\mathrm{Cs}_{2} \mathrm{CuBr}_{4}$ & $1 / 2$ & 2.44 & 0.38 & $16.1 \mathrm{~K}$ & 1.13 & 0.20 & 2.86 & [34] \\
\hline $\mathrm{NiGa}_{2} \mathrm{~S}_{4}$ & 1 & $1^{\mathrm{a}}$ & -0.75 & $6.45 \mathrm{~K}$ & - & - & - & [9] \\
\hline $\mathrm{PdCrO}_{2}$ & $3 / 2$ & 1 & 0.25 & $42.7 \mathrm{~K}^{\mathrm{b}}$ & $4 / 3$ & $0.826(0.666)$ & 1 & 35,36 \\
\hline
\end{tabular}

a antiferromagnetic next-nearest neighbor interaction $J_{3}>\left|J_{1}\right|,\left|J_{2}\right|$ dominates[9]

${ }^{\mathrm{b}}$ from $v_{x}=v_{y}=3200 \mathrm{~m} / \mathrm{s}$

therefore the (short dashed) $E_{c l}(\phi)$ curve for the columnar AF doubles its amplitude to $-2 J_{c} \sin \phi=-2 J_{2}$ and now lies below the spiral phase in the above range of $\phi$, thus leading to a columnar AF ground state for the square lattice model. This explains the main difference of the square lattice and triangular classical phase diagrams. The latter is shown in Fig. 4

It is naturally important to investigate how many features of the classical phase diagram are modified or changed radically by the effect of quantum fluctuations. This relates only to the AF and spiral phases since in the FM phase quantum fluctuations are absent. We have included their effect in order $(1 / S)$ within conventional LSW approximation. It must be clearly stressed that the latter breaks down when there is no long range magnetic order. This happens in possibly three regions of the phase diagram: At the boundaries of the spiral region to the AF and FM phase and in the center of it $\left(J_{1}=0\right)$ where decoupled chains lead lead to a quasi-1D spin liquid state to be discussed further below.

First we note that, as in the square lattice model the phase boundaries are unchanged in LSW approach when zero point fluctuations are included in the ground state energy. However, in the triangular model we have the additional aspect that the spiral magnetic structure itself, i.e., the spiral propagation vector $\mathbf{Q}$ is modified by the zero point contributions to the ground state energy as derived in Sec. IV Fig. 55.
More pronounced quantum effects occur in the the ground state energy which is shown in Fig.66. The quantum corrected $E_{g s}$ curve (full line) lies considerably below the classical on one (dashed line) in the AF and spiral region (as already mentioned there are no corrections in the FM state). In particular we note that even for the $1 \mathrm{D}$ case $\left(\phi / \pi=0.5\right.$ or $\left.J_{1}=0\right)$ where nominally spin wave theory is not valid we obtain an excellent value as compared to the exact Bethe ansatz result [29] energy which is only slightly lower. This may be justified by the fact that although no strict long range order exists quasi-long range order signified by an algebraic decay of the correlation function is present in quasi-1D AF spin chains. Therefore the longwavelength spin excitations which are most important for the zero point corrections are qualitatively reasonably described by spin waves.

Now we summarize the most important quantum effects concerning the renormalization of the ordered moment $m_{Q}$ that have been derived in Sec. VI (Fig. $7 \mathrm{k}$ ). For comparison we also present the previous results for the $J_{1}-J_{2}$ square lattice model in Fig. 77p. In Fig. $7 \mathrm{p}$ in the AF regime the variation is smooth starting at the classical value $m_{Q} /\left(\mu_{B} S\right)=0.5$ at the FM boundary and passing through the unfrustrated (square lattice) value of $m_{Q} /\left(\mu_{B} S\right)=0.3$ at $\phi=0\left(J_{2}=0\right)$ and vanishing logarithmically at the spiral phase boundary point $\left(\phi / \pi \approx 0.15\right.$ or $\left.J_{2}=0.5 J_{1}\right)$. For larger $\phi$ in the spiral 
region the moment recovers and reaches a maximum value of $m_{Q} /\left(\mu_{B} S\right)=0.24$ in the frustrated isotropic triangular model $\left(\phi / \pi=0.25\right.$ or $\left.J_{1}=J_{2}\right)$. This is in reasonable agreement with more exact DMRG results [5] giving $m_{Q} /\left(\mu_{B} S\right) \simeq 0.20$. Then $m_{Q}$ decreases rapidly with increasing $\phi$ and is destroyed in a finite region around the 1D chain model $\left(\phi / \pi=0.5\right.$ or $\left.J_{1}=0\right)$ signifying the onset of a quasi-1D spin liquid state. The width of the sector where $m_{Q}$ vanishes in LSW approximation is given by the shaded area in Fig. 4 . For still larger $\phi$ the ordered moment $m_{Q}$ is stabilized again and reaches the classical value at the FM boundary.

The effect of quantum fluctuations on the ordered moment can be tuned by applying a magnetic field. A non-monotonic behavior as function of $H$ was found in Fig. 8due to reduction of zero point fluctuations by the polarization, quite similar to the $J_{1}-J_{2}$ model [32]. In contrast, however, the triangular model also exhibits a non-monotonic behavior of the zerofield moment $m_{Q}(H=0, \phi)$ as function of control parameter $\phi$ around the isotropic point $(\triangle)$ with $\phi / \pi=0.25$ as seen in Fig. 8 .

The homogeneous magnetization and the susceptibility for the triangular model were found to be very anomalous in the spiral phase (Figs. 910). LSW approximation breaks down for these quantities in more than half of the spiral sector $0.4 \leq \phi / \pi \leq 0.85$. It is remarkable that the homogeneous magnetization $\mathrm{M}$ (and $\chi$ ) is not recovered close to the FM sector even though the the ordered moment is stabilized again beyond the 1D point ( $\|$ ) for $\phi / \pi>0.6$ (Fig. 7 ).

Thus in LSW treatment there are three instability regions of magnetic order in the anisotropic triangular model of Fig. 7 p. 1) At the AF-spiral phase boundary point. In LSW approach we cannot identify a sizable finite sector size of the instability. This is in contrast to the square lattice $J_{1}-J_{2}$ model (Fig. 7p) where the instability for $J_{1}=J_{2}$ occurs in a finite sector [15]. The true ground state in that sector is likely of the nonmagnetic staggered dimer type [37] and this has also been suggested for the triangular model from finite temperature series expansions [21]. However in this case a finite sector for the nonmagnetic state shifted to slightly larger $\phi / \pi \simeq 0.19$ is predicted. This is supported by modified spin wave theory and exact diagonalization results [24]. 2) The quasi -1D spin fluctuations around $\phi / \pi \simeq 0.5$ lead to an extended region of a quasi-1D spin liquid state with algebraic spin correlations. This is opposite to the situation in the square lattice $J_{1}-J_{2}$ model which achieves the unfrustrated 2D HAF value $m_{Q} /\left(\mu_{B}\right)=0.3$ at this point due to decoupled unfrustrated $\left(\mathrm{J}_{2}\right.$ only) sublattices. 3 ) at the spiral-FM phase boundary the staggered moment $m_{Q}$ is stable, however homogeneous magnetization and susceptibility behave very anomalous indicating again a tendency to instability. This behavior of $M$ and $\chi$ is similar to the square lattice $J_{1}-J_{2}$ model, although there the instability range in $\phi$ is smaller. On the other hand for the square lattice model the ordered moment itself becomes unstable at the equivalent columnar AF-FM boundary (Fig. 7p). In this case it is known that a non-magnetic spin-nematic phase [38] is established in this regime.

As the main purpose of this work is to give a survey of ground states and excitations for all triangular anisotropies we now further discuss the variation of the excitation spectrum in LSW theory for the full range of $\phi$. In each of the magnetically ordered sectors we choose three typical values of $\phi$ for the 2D spin wave dispersion and present it both as 3D topographic plot and in shaded contours in the $q_{x}-q_{y}$ plane.

In Fig. 11 we present it for the ferromagnetic regime. In the underlying contour plot the dark regions represent locations in the BZ where the spin wave energy is low and the bright (yellow) regions correspond to high energies. Apparently when $\phi$ approaches the spiral phase boundary the BZ regions with low energy excitations become large. This continues into the adjacent spiral phase and may play a role in the extended instability of the low-field magnetization or susceptibility discussed above.

The AF spin wave dispersions are shown in In Fig. 12. They exhibit a continuous change of anisotropy characteristics as function of $\phi$. Deep inside the AF phase (a) and for the square lattice case (b) the low energy spin waves are centered around zone center and zone boundary points in the BZ whereas at the border to the spiral phase they are located in large overlapping zone boundary regions (c).

The results for the spiral phase are presented in Fig. 14. The low energy spin waves are now located around incommensurate ordering wave vectors $\mathbf{Q}$ in the $\mathrm{BZ}$ indicated by white arrows. Of particular interest is the case (b) with $J_{2} / J_{1} \approx 3$ or $\phi / \pi \approx 0.4$ which is close to the anisotropy ratios of $\mathrm{Cs}_{2} \mathrm{CuCl}_{4}$ and $\mathrm{Cs}_{2} \mathrm{CuBr}_{4}$ (Table II). In this case overlapping regions of low energy spin waves along $q_{y}$ direction indicate a large renormalization of the moment. Indeed from Fig. 7 one concludes a moment reduction to $m_{Q} /\left(\mu_{B} S\right)=0.043$ in LSW approximation. Thus $\mathrm{Cs}_{2} \mathrm{CuCl}_{4}$ is at the very limit of applicability of spin wave theory [18, 19]. A slight increase of $\phi$ would push it to the nonmagnetic quasi-1D spin liquid regime. Of course this discussion is oversimplified as in the real compound there are further interactions like a DzyaloshinskiiMoriya term and inter-plane exchange [10]. The latter has the tendency to stabilize the magnetic order. Beyond the quasi 1D spin liquid state the ordered moment is reestablished (Fig. 7). The spin wave dispersion in this regime (c) has however still large $\mathbf{q}$ space regions with small energies. This may be the reason why the homogeneous magnetization and susceptibility is not stabilized again (Fig. 9).

Finally we discuss the relation of our survey using LSW theory to other analytical and numerical results. This is confined to the sector of $\mathrm{AF} / \mathrm{spiral}$ phases studied before whose existence is predicted by all methods. The precise values for $\phi$ at which the phase boundaries occur and the position and size of nonmagnetic sectors, however, depends on the method used.

In LSW theory the results are simple because magnetic phase boundaries are identical to the classical ones (Table I). In particular the AF/spiral boundary is at $\phi=\tan ^{-1}(1 / 2) \approx$ $0.15 \pi$ and the ordered moment is vanishing only at this point (i.e. there is no finite size of a nonmagnetic sector in Fig. 7 as opposed to the square lattice model in Fig. 7p at the same value of $\phi$ ). The spiral sector extends up to $\phi / \pi \approx 0.42$ where the moment vanishes due to the appearance of the quasi-1D spin liquid. 
In modified spin wave theory (MSW) [23, 24] the AF phase is stabilized to larger values of $\phi / \pi \approx 0.20$ where, in contrast to LSW theory the nonmagnetic state becomes stable in a finite interval up to $\phi / \pi \approx 0.23$. It is followed by the spiral phase which achieves its maximum staggered moment $m_{\mathbf{Q}} /\left(\mu_{\mathrm{B}} S\right) \approx 0.685$ for the isotropic case $\phi / \pi=0.25$ with the $120^{\circ}$ structure, as in LSW theory. The latter has $m_{\mathbf{Q}} /\left(\mu_{\mathrm{B}} S\right) \approx 0.478$, in better agreement with the DMRG value $m_{\mathbf{Q}} /\left(\mu_{\mathrm{B}} S\right) \approx 0.41$. The spiral magnetic phase then is stable only in a comparatively small interval up to $\phi / \pi \approx 0.32$ where already the quasi-1D spin liquid appears. Thus for the exchange anisotropy commonly used for $\mathrm{Cs}_{2} \mathrm{CuCl}_{4}$ (Table III) MSW theory predicts this compound would have a nonmagnetic ground state in a purely 2D model [24].

In addition the AF/spiral boundary region has been studied before using a numerical dimer series expansion technique [21]. Here also the AF phase is stabilized to larger values $\phi / \pi \approx 0.19$ followed by a finite dimer spin liquid phase interval up to a value $\phi / \pi \approx 0.24$ obtained from vanishing staggered moment or spin gap, respectively. For larger $\phi$ values the spiral phase is again stabilized, however the maximum moment $m_{\mathbf{Q}} /\left(\mu_{\mathrm{B}} S\right) \approx 0.18$ is achieved only for an incommensurate spiral phase with $\phi / \pi \approx 0.34$, considerably larger than in LSW or MSW theory where it occurs for the $120^{\circ}$ structure of the isotropic case with $\phi / \pi=0.25$. The quasi-1D spin liquid boundary is difficult to determine with this method, but the staggered moment vanishes at similar values $\phi / \pi \approx 0.44$ comparable to the LSW result.

Thus from these investigations beyond LSW theory one concludes the following general trends in the AF/spiral region: The stability of the AF phase is pushed to larger $\phi$ and a finite spin liquid interval appears between $\phi / \pi \approx 0.19 \ldots 0.20$ and $\phi / \pi \approx 0.23 \ldots 0.24$. In addition MSW predicts an earlier onset of the quasi-1D spin liquid regime. Thus the stability region of the spiral magnetic phase generally shrinks as compared to the LSW case. In particular for the exchange ratio commonly used for $\mathrm{Cs}_{2} \mathrm{CuCl}_{4}$ (corresponding to $\phi / \pi=0.41)$ LSW theory and dimer series expansion still lead to a marginally ordered spiral magnetic state whereas MSW theory predicts already a disordered state for the purely $2 \mathrm{D}$ model without interlayer coupling.

\section{SUMMARY AND CONCLUSION}

In this work we have given a global survey of the effect of quantum fluctuations in triangular spin lattices with anisotropic ferro-and antiferromagnetic exchange.

We have explored the phase diagram of the triangular $J_{1^{-}}$ $J_{2}$ model in the full range of the control parameter $\phi=$ $\tan ^{-1}\left(J_{2} / J_{1}\right)$ using the linear spin wave method. The contribution of zero point fluctuations to ground state energy and wave vector, ordered moment, magnetization and susceptibility has been investigated. In particular we point out that the ordered moment shows non-monotonic dependence both on the field and on the control parameter $\phi$ close to the isotropic triangular system. We also made a systematic comparison with the related square lattice $J_{1}-J_{2}$ model concerning the possible phases and their stability as well as their ordered moment reduction by quantum fluctuations.

Furthermore we discussed the characteristic anisotropies of spin wave dispersion as the control parameter $\phi$ is tuned through the phase diagram. The instability of the ordered moment for the AF/spiral boundary and the quasi-1D 'spin liquid' region is found to be associated with low energy modes in large areas of the BZ. The spin wave velocities as function of $\phi$ are very anisotropic, in particular in the spiral phase. This may be used for a determination of the exchange ratio. A further diagnostic tool fur this purpose is the (low-) field dependence of the ordered moment which was found to depend strongly on the control parameter $\phi$. Naturally the LSW method used here is not suitable to discuss the region of moment instability or spin-liquid regions. More sophisticated numerical and analytical methods as mentioned in the introduction have to be use for this purpose.
[1] T. Jolicoeur and J. C. Le Guillou, Phys. Rev. B 40, 2727 (1989)

[2] A. V. Chubukov, S. Sachdev, and T. Senthil, J. Phys.: Condens. Matter 6, 8891 (1994).

[3] H. Kawamura and S. Miyashita, J. Phys. Soc. Jpn. 53, 4138 (1984)

[4] B. Bernu, P. Lecheminant, C. Lhuillier, and L. Pierre, Phys. Rev. B 50, 10048 (1994)

[5] S. R. White and A. L. Chernyshev, Phys. Rev. Lett. 99, 127004 (2007)

[6] O. A. Starykh, A. V. Chubukov, and A. G. Abanov, Phys. Rev. B 74, 180403 (2006)

[7] A. L. Chernyshev and M. E. Zhitomirsky, Phys. Rev. B 79, $144416(2009)$

[8] W. Zheng, J. O. Fjærestad, R. R. P. Singh, R. H. McKenzie, and R. Coldea, Phys. Rev. B 74, 224420 (2006)

[9] S. Nakatsuji, Y. Nambu, and S. Onoda, J. Phys. Soc. Jpn. 79, 011003 (2010)

[10] R. Coldea, D. A. Tennant, K. Habicht, P. Smeibidl, C. Wolters, and Z. Tylczynski, Phys. Rev. Lett. 88, 137203 (2002)

[11] R. Coldea, D. A. Tennant, and Z. Tylczynski, Phys. Rev. B 68, 134424 (2003)

[12] S. Zvyagin, D. Kamenskyi, M. Ozerov, J. Wosnitza, M. Ikeda, T. Fujita, M. Hagiwara, A. Smirnov, T. Soldatov, A. Shapiro, J. Krzystek, R. Hu, H. Ryu, C. Petrovic, and M. Zhitomirsky, Phys. Rev. Lett. 112, 077206 (2014)

[13] T. Ono, H. Tanaka, H. Aruga Katori, F. Ishikawa, H. Mitamura, and T. Goto, Phys. Rev. B 67, 104431 (2003)

[14] P. T. Cong, B. Wolf, M. de Souza, N. Krüger, A. A. Haghighi$\mathrm{rad}$, S. Gottlieb-Schoenmeyer, F. Ritter, W. Assmus, I. Opahle, K. Foyevtsova, H. O. Jeschke, R. Valentí, L. Wiehl, and M. Lang, Phys. Rev. B 83, 064425 (2011)

[15] B. Schmidt, M. Siahatgar, and P. Thalmeier, Phys. Rev. B 81, 165101 (2010)

[16] J. Merino, R. H. McKenzie, J. B. Marston, and C. H. Chung, J. Phys.: Condens. Matter 11, 2965 (1999).

[17] A. E. Trumper, Phys. Rev. B 60, 2987 (1999) 
[18] M. Y. Veillette, J. T. Chalker, and R. Coldea, Phys. Rev. B 71, $214426(2005)$

[19] M. Y. Veillette, A. J. A. James, and F. H. L. Essler, Phys. Rev. B 72, 134429 (2005)

[20] J. Reuther and R. Thomale, Phys. Rev. B 83, 024402 (2011)

[21] Z. Weihong, R. H. McKenzie, and R. R. P. Singh, Phys. Rev. B 59, 14367 (1999)

[22] M. Q. Weng, D. N. Sheng, Z. Y. Weng, and R. J. Bursill, Phys. Rev. B 74, 012407 (2006)

[23] P. Hauke, T. Roscilde, V. Murg, J. I. Cirac, and R. Schmied, New J. Phys. 13, 075017 (2011)

[24] P. Hauke, Phys. Rev. B 87, 014415 (2013)

[25] R. L. Doretto and M. Vojta, Phys. Rev. B 85, 104416 (2012).

[26] N. Shannon, B. Schmidt, K. Penc, and P. Thalmeier, Eur. Phys. J. B 38, 599 (2004)

[27] A. A. Tsirlin, B. Schmidt, Y. Skourski, R. Nath, C. Geibel, and H. Rosner, Phys. Rev. B 80, 132407 (2009)

[28] T. Nikuni and H. Shiba, J. Phys. Soc. Jpn. 62, 3268 (1993)

[29] H. Bethe, Z. Phys. 71, 205 (1931).
[30] L. Hulthén, Arkiv för Matematik, Astronomi och Fysik 26A, 1 (1938).

[31] I. Affleck, J. Phys. A: Math. Gen. 31, 4573 (1998)

[32] M. Siahatgar, B. Schmidt, and P. Thalmeier, Phys. Rev. B 84, 064431 (2011)

[33] A. Honecker, J. Phys.: Condens. Matter 11, 4697 (1999)

[34] S. Zvyagin, M. Ozerov, D. Kamenskyi, J. Wosnitza, M. Ikeda, T. Fujita, M. Hagiwara, J. Krzystek, R. Hu, H. Ryu, and C. Petrovic, arXiv:1306.3887 (2013).

[35] H. Takatsu, H. Yoshizawa, S. Yonezawa, and Y. Maeno, Phys. Rev. B 79, 104424 (2009)

[36] M. Mekata, T. Sugino, A. Oohara, Y. Oohara, and H. Yoshizawa, Physica B 213-214, 221 (1995)

[37] R. R. P. Singh, W. Zheng, C. J. Hamer, and J. Oitmaa, Phys. Rev. B 60, 7278 (1999)

[38] N. Shannon, T. Momoi, and P. Sindzingre, Phys. Rev. Lett. 96, 027213 (2006) 